\title{
Signatures of human skin in the millimetre wave band (80-100) $\mathbf{G H z}$
}

\author{
Amani Y. Owda*, Nacer-Ddine Rezgui, Neil A. Salmon \\ School of Engineering, Manchester Metropolitan University, M1 5GD, United Kingdom
}

\begin{abstract}
With the performance of millimeter wave security screening imagers improving (reduced speckle, greater sensitivity, and better spatial resolution) attention is turning to identification of anomalies which appear on the human body. Key to this identification is the understanding of how the emissive and reflective properties vary over the human body and between different categories of people, defined by age and gender for example. As the interaction of millimetre waves with the human body is only a fraction of a millimetre into the skin, precise measurement of the emission and reflection of this radiation will allow comparisons with the norm for that region of the body and person category. On an automated basis at security screening portals, this will increase detection probabilities and reduce false alarm rates, ensuring high throughputs at entrances to future airport departure lounges and transport networks.
\end{abstract}

A technique to measure the human skin emissivity in vivo over the frequency band $80 \mathrm{GHz}$ to $100 \mathrm{GHz}$ is described. The emissivities of the skin of a sample of 60 healthy participants (36 males and 24 females) measured using a $90 \mathrm{GHz}$ calibrated radiometer was found to range from $0.17 \pm 0.002$ to $0.68 \pm 0.002$. The radiometric measurements were made at four locations on the arm, namely: palm of hand, back of hand, dorsal surface of the forearm, and volar side of the forearm, where the water content and the skin thickness are known to be different. These measurements show significant variation in emissivity from person to person and, more importantly, significant variation at different locations on the arms of individuals. Males were found to have an emissivity 0.03 higher than those of females. The emissivity of the back of the hand, where the skin is thinner and the blood vessels are closer to the skin surface, was found to be lower by 0.0681 than the emissivity of the palm of the hand, where the skin is thicker. The measurements also show that the emissivity of the volar side location where the blood vessels are closer to the skin surface is lower by 0.0677 than the emissivity of the dorsal surface location. The measured differences agree with those differences estimated by a half space electromagnetic model of the interaction and can be interpreted in terms of the differing water contents and skin thickness of those regions of the body.

Keywords: security screening, emissivity, millimetre waves, permittivity, skin thickness, water content.

\section{INTRODUCTION}

As passive millimetre wave imaging is free from artefacts such as speckle and sensitivity to the alignment, it provides a means to screen people for concealed threats through the clothing down to the skin, for all regions of the human body [1]. In this paper, attention is turning to identification of anomalies, which appear on the human body and can be identified by measuring the signature of the human skin at the millimeter wave frequency band. Measurement and validation of the emissivity of human skin at MMW frequency bands is an essential requirement, to help assess the feasibility of increasing the detection probabilities and reducing the false alarm rate when screening at entrances to airport departures areas. The main advantages of the passive millimeter wave imaging system are: 1) human skin signature can be measured without exposing the human body to any type of radiation, 2) radiometric sensitivity is sufficient to sense different surfaces attached to the human skin such as liquid and metallic objects, and 3) the measurements and processing of data can be made in tens of seconds by using a non-contact sensor with high precision in which this will minimise the systematic uncertainty.

*amaniabubaha@gmail.com; Manchester Metropolitan University; mobile number +44 7402939756. 


\section{THE SYSTEM}

\subsection{Experimental setup}

A radiometer sensitive over the frequency band $80-100 \mathrm{GHz}$ was used for measuring the human skin emissivity in vivo. The experimental setup for measurement comprises; a horn antenna connected to a radiometer that consists of a twostage low-noise amplifier (LNA), zero bias detector and buffer amplifier. The radiometer is connected through coaxial cable to a digital voltmeter and through wires to a DC power supply, as illustrated in Figure 1 . The horn antenna has a rectangular aperture $\left(30 \times 25 \mathrm{~mm}^{2}\right)$ and a nominal gain of $20 \mathrm{dBi}$ over the frequency band $(80-100) \mathrm{GHz}$.

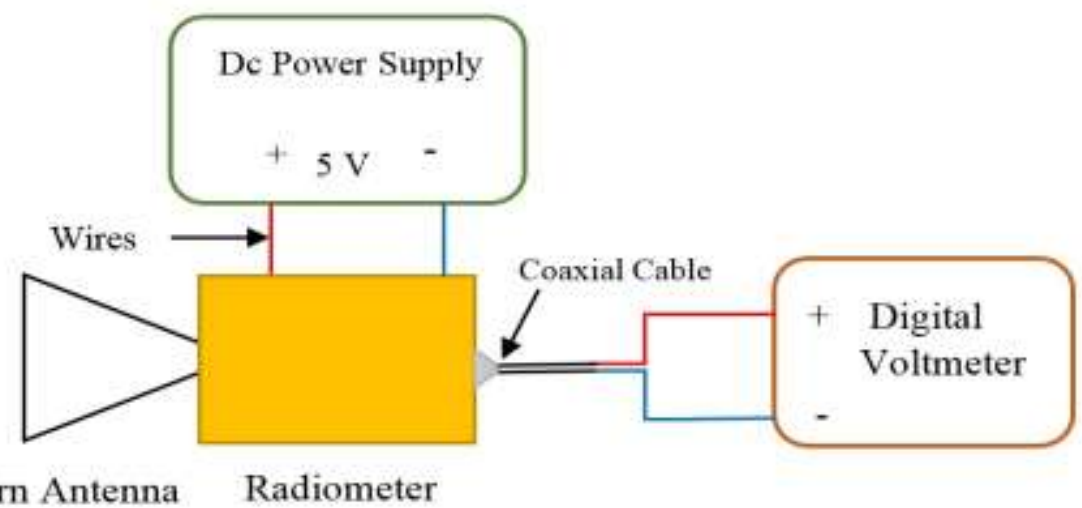

Figure 1. Experimental setup for measuring the human skin emissivity over the frequency band from $80 \mathrm{GHz}$ to $100 \mathrm{GHz}$

\subsection{Radiometric calibration and testing}

The radiometer was calibrated using liquid nitrogen and ambient temperature sources, as illustrated in Figure 2 . The horn antenna of the radiometer may be placed at distance $(\sim 5.0 \mathrm{~cm})$ from two different radiation sources located in the same plane: "Hot" black body (ambient temperature source calibration). A piece of carbon loaded foam absorber with $\mathrm{T}_{\mathrm{H}}=$ Tambient $=293 \mathrm{~K}$, and "Cold" black body (liquid nitrogen source calibration). A piece of carbon loaded foam absorber was dipped in liquid nitrogen at $\mathrm{T}_{\mathrm{C}}=77 \mathrm{~K}$. The cold load calibration measurements were taken within 5 seconds or less before the liquid nitrogen evaporates. Foam absorbers had a rectangular shape and emissivity values greater than 0.99 over the frequency band $80-100 \mathrm{GHz}$, thus they behave as good approximations to a black body emitter. The difference in temperature between the hot and the cold load is $\sim 216 \mathrm{~K}$. This large difference is important to provide an accurate calibration. The calibration Y-factor, defined as the ratio of receiver output when measuring the hot black body source, to that measuring the cold source was 1.408 . This gives a receiver noise temperature of $453.7 \mathrm{~K}$ and noise figure of 2.55. These measurements were taken from ten separate experiments and at each experiment, the calibration measurements were repeated 5-10 times so the device was stable and the measurements were consistent.
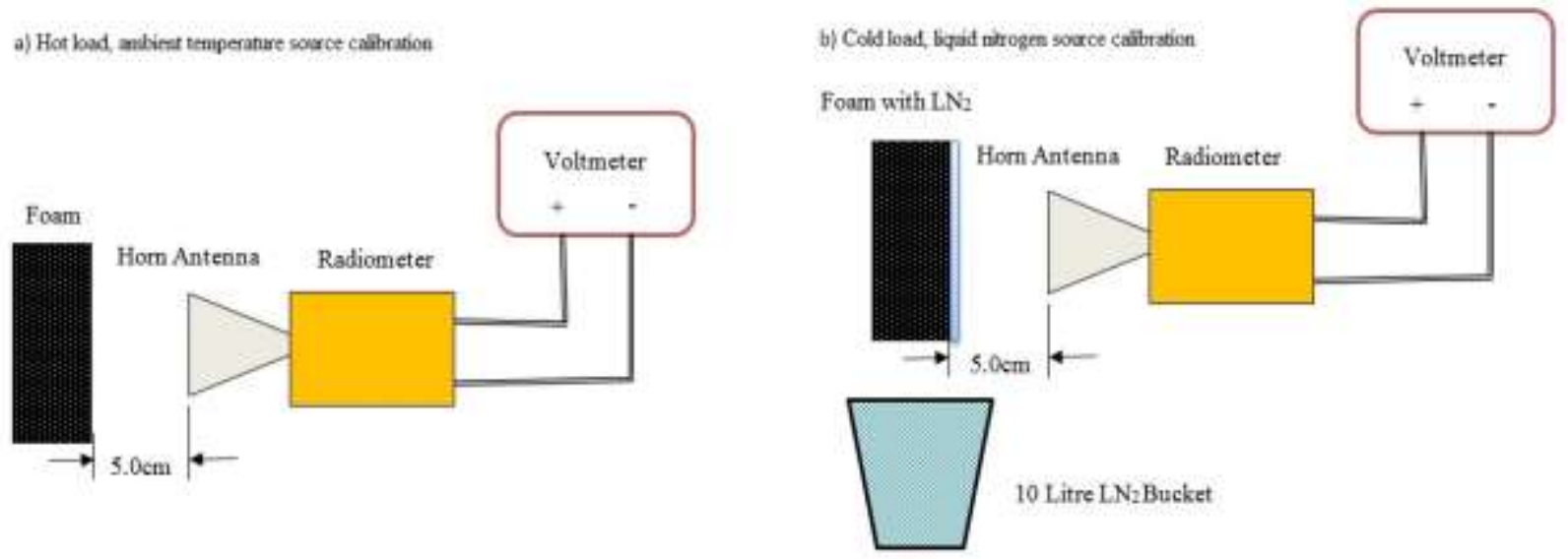

Figure 2. Radiometric calibration using ambient temperature source (hot load) and liquid nitrogen source (cold load) 
The amount of self-emission reflected back from subjects was investigated by moving a metal plate in a distance $1.0 \mathrm{~cm}$ from the horn antenna beam. The mean level of self-emission reflected back from the metal plate (100\% reflective surface) was measured to be in the range of $294-295 \mathrm{~K}$ with standard deviation $\pm 1.0 \mathrm{~K}$. The results presented show that the radiation temperature from the metal plate is approximately the same as the ambient temperature, and therefore no parasitic signals were identified when emission from the receiver was reflected back into the system by a normal incidence metal reflector placed directly in front of the horn antenna.

Millimeter-Wave emission emitted from a fluorescent light located $5.0 \mathrm{~cm}$ from the horn antenna was found to increase the radiation temperature measured by the radiometer by an amount $62-74 \mathrm{~K}$; a mean value of $67.5 \mathrm{~K}$ with a standard deviation of $\pm 4.0 \mathrm{~K}$. When the fluorescent light was located directly in the beam of the horn antenna, the radiation temperature was found to increase by an amount $80-100 \mathrm{~K}$; a mean value of $84.3 \mathrm{~K}$ with a standard deviation of $\pm 8.0 \mathrm{~K}$. This effect was cancelled during the experimental work by turning off all the fluorescent light in the lab [2].

\section{METHODOLOGY OF MEASURING HUMAN SKIN EMISSIVITY}

The complete system except for an opening for the subject to be measured (skin) was enclosed in an anechoic region made by surrounding the majority of the radiometer and antenna with carbon loaded absorbing foam, as illustrated in Figure 3. This prevented radiation from external sources, be it from the outdoors or other people in the environment, getting into the system to corrupt signals.

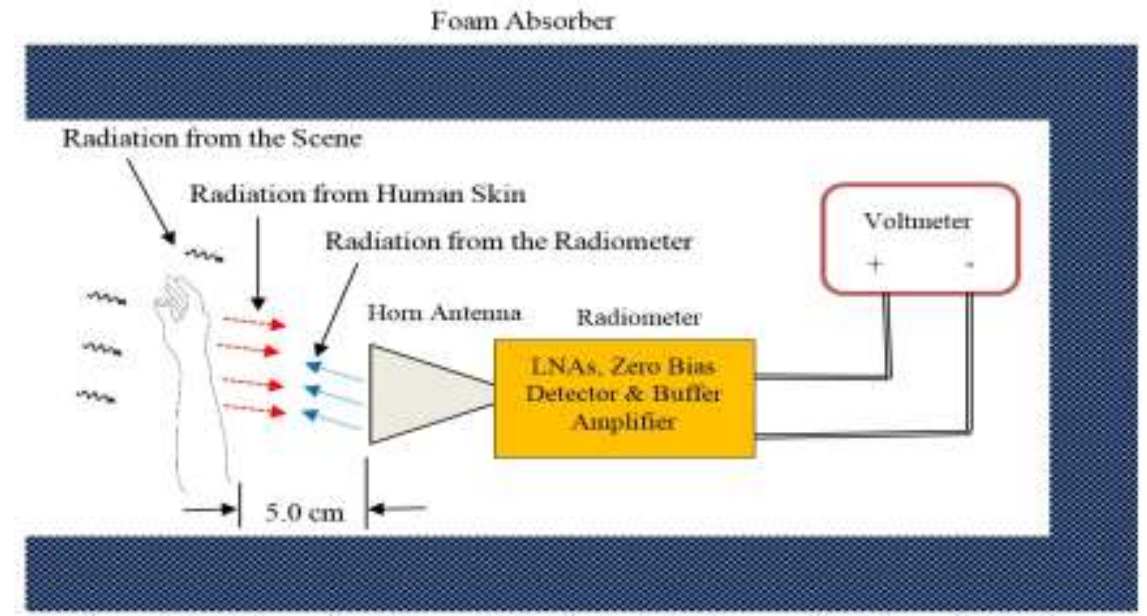

Figure 3. Experimental description for in vivo radiometric emissivity measurements performed on human hand

The target area of the skin was located $5.0 \mathrm{~cm}$ from the horn antenna of the radiometer, and the measurements were done indoors in an anechoic environment, and therefore the system response is assumed to be linear. Under these circumstances, the output voltage when measuring the liquid nitrogen and ambient temperature calibration sources can be expressed in terms of the receiver noise temperature $\mathrm{T}_{\mathrm{N}}$ in $\mathrm{K}$, and the receiver responsivity, $\alpha$ in $\mathrm{V} / \mathrm{K}$ [3]:

$$
\begin{aligned}
& V_{C}=\alpha\left(T_{C}+T_{N}\right) \\
& V_{H}=\alpha\left(T_{H}+T_{N}\right)
\end{aligned}
$$

From Equations (1) and (2) the receiver responsivity, $\alpha$, and the emissivity of the skin, $\eta$, are [3]:

$$
\begin{aligned}
& \alpha=\frac{\left(V_{H}-V_{C}\right)}{\left(T_{H}-T_{C}\right)} \\
& \eta=\frac{\left(V_{S}-V_{H}\right)\left(T_{H}-T_{C}\right)}{\left(V_{H}-V_{C}\right)\left(T_{S}-T_{H}\right)}
\end{aligned}
$$


An infrared thermometer with an absolute measurement uncertainty of $\pm 1.5{ }^{\circ} \mathrm{C}$ was used to measure the thermodynamic temperatures of the skin, $\mathrm{T}_{\mathrm{S}}$ directly before and after the measurements. A digital voltmeter with a precision of $0.1 \mathrm{mV}$ was used to measure the output voltage for the target area of the skin; Vs. Error propagation through Equation (4) indicates the uncertainty on the measured emissivity is \pm 0.002 .

\section{RESULTS}

The objective of these experiments is to study how the signature of the human skin varies between individuals, gender and locations over the frequency band 80-100 GHz. Emissivity measurements were performed on 60 healthy participants; 36 males and 24 females having a variety of ethnicities, ages, and body mass index. The measurements were made at four locations on the body and these were: 1) palm of hand, 2) back of hand, 3) dorsal surface of the forearm, and 4) volar side of the forearm. These locations were chosen due to variation in skin thickness and water content.

\subsection{Male emissivity measurements}

Measurements of human skin emissivity of a sample of 36 male participants are presented in Figure 4. The measurements show variation in emissivity between individuals and locations on the arm. These variations are due to the skin thickness and blood vessels (water content) that varies from location to location across the human body and between individuals [4]. The emissivity from male sample was found to range from $0.18 \pm 0.002$ to $0.68 \pm 0.002$, with mean $(\mu)$ and standard deviation $(\sigma)$ for all measurements location being 0.416 and 0.091 respectively. In general, lower values of emissivity are results of measuring particularly thin skin on the volar side and back of hand [5], whereas higher values of emissivity are results of measuring thick skin on the dorsal surface and palm of hand [6].

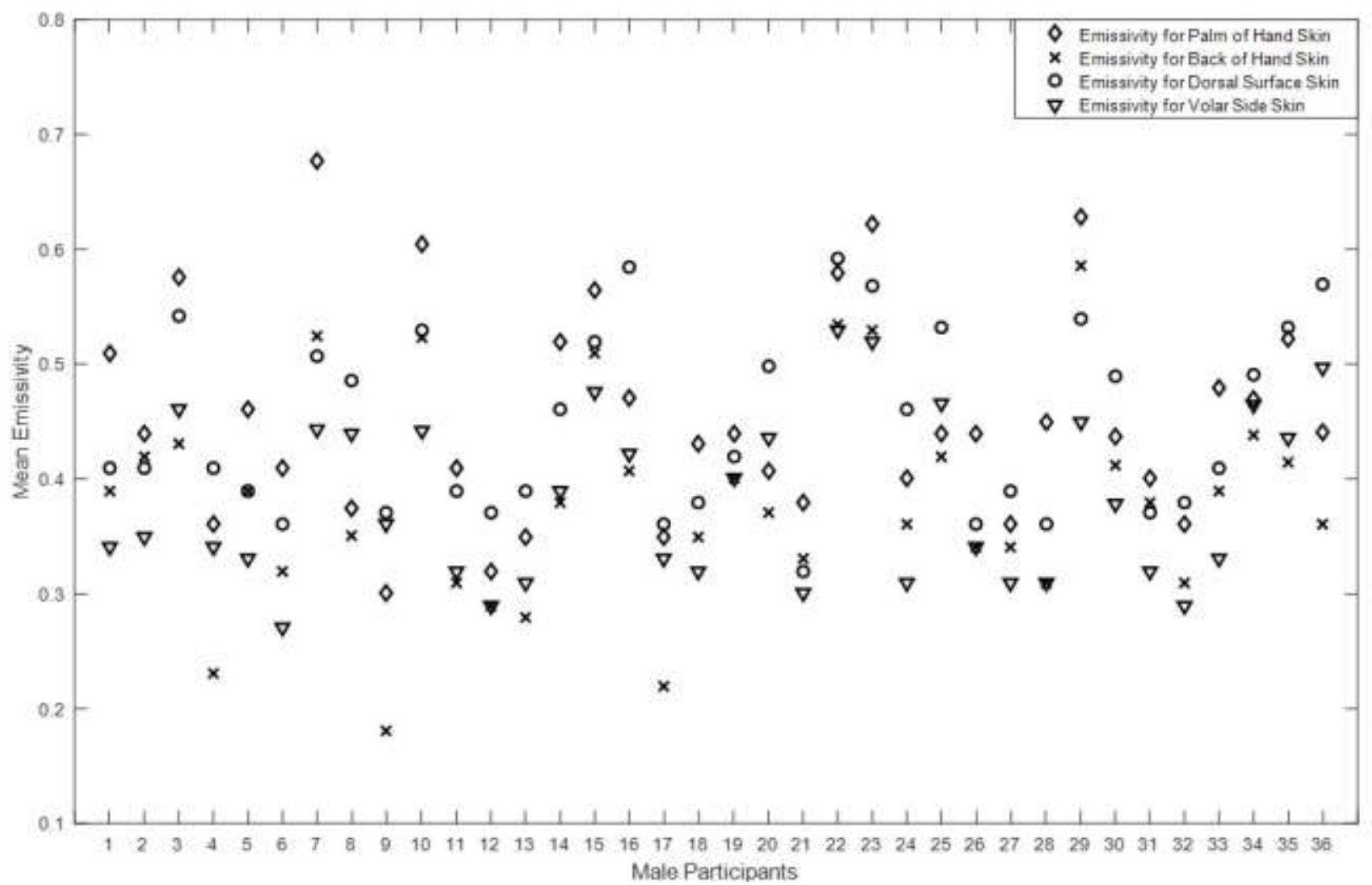

Figure 4. Radiometric emissivity measurements for 36 male participants performed over the frequency band $80-100 \mathrm{GHz}$ 
The measurements indicate the differences in emissivities between thicker regions of skin (dorsal forearm and palm of hand) and thinner regions of skin (volar forearm and back of hand) are in the range from 0.068 to 0.074 with a sample standard deviation in the range of 0.032 to 0.041 respectively. These results show a clear signature for the human skin emissivity at the millimeter wave frequency band (80-100) GHz. The presented signature might be useful for identifying anomalies on the human body by comparing the measured emissivity of the subject area of the skin with the standard values of the population (mean emissivity \pm standard deviation), so any value much higher or much lower than the standard values might identify anomalies.

\subsection{Female emissivity measurements}

Measurements of human skin emissivity in a sample of 24 female participants are presented in Figure 5. Emissivity for the dorsal surface and palm of hand skin are higher than volar side and back of hand skin. Variation in emissivity between individuals and locations on the hand are consistent with the variations in male participants. However, female emissivity was found to be lower than that of male in the range from $0.17 \pm 0.002$ to $0.587 \pm 0.002$, with mean and standard deviation for all measurement locations being 0.383 and 0.0839 respectively. The measurements also indicate the differences in emissivity values between thicker regions of skin and thinner regions of skin are in the range from 0.0597 to 0.0679 with a sample standard deviation in the range of 0.0358 to 0.0439 .

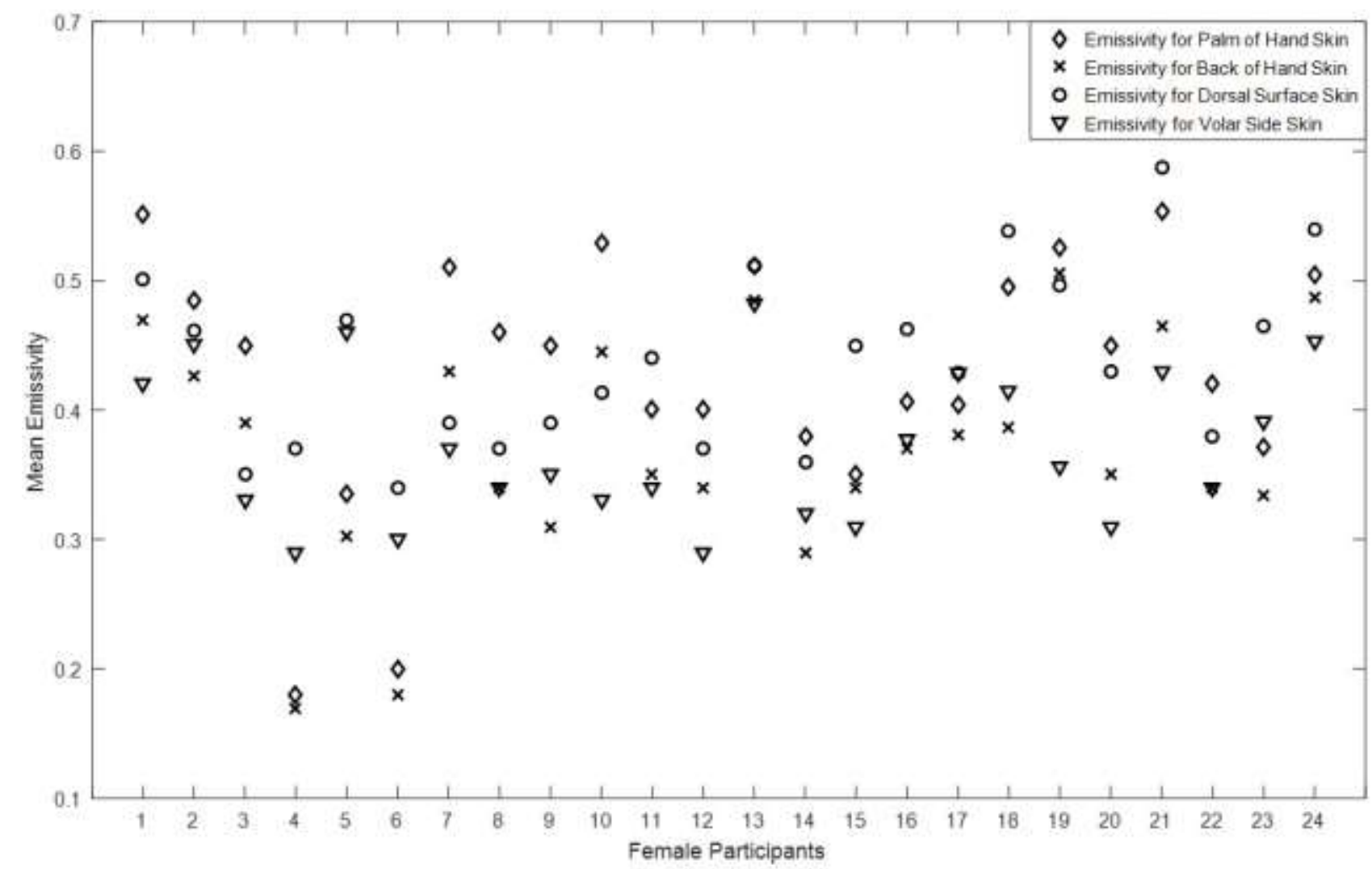

Figure 5. Emissivity measurements for 24 female participants performed over the frequency band $80-100 \mathrm{GHz}$ 


\subsection{Comparison between male and female emissivity}

Experimental measurements of human skin emissivity of 36 male and 24 female healthy participants are presented in Table 1. The measurements show that emissivities of male participants are higher than that of female participant's at all locations on the arm. This finding is consistent with the fact that male skin is thicker than that of female skin [7]. Estimating the sample mean emissivity values for the male and female participants separately indicates the difference between male and female emissivity is $\sim 0.03$. This difference is $\sim 15$ times the systematic measurement uncertainty $( \pm 0.002)$ indicating a significant difference in the emissivity between male and female participants. Furthermore, the measurements show that for both genders, the emissivity is higher in the locations where the skin is thick: dorsal surface of the forearm and palm of hand [6], and it is lower in the locations where the skin is thin: volar side of the forearm and back of hand skin [5]. These measurements show strong correlation between human skin emissivity and skin thickness.

Table 1. Comparison in emissivity, between male and female participants at four locations on the arm.

\begin{tabular}{|c|c|c|c|c|}
\hline Location & $\begin{array}{c}\text { Mean Emissivity } \\
\text { Male }\end{array}$ & $\begin{array}{c}\text { Standard } \\
\text { Deviation (M) }\end{array}$ & $\begin{array}{c}\text { Mean Emissivity } \\
\text { Female }\end{array}$ & $\begin{array}{c}\text { Standard } \\
\text { Deviation (F) }\end{array}$ \\
\hline Palm of Hand & 0.4511 & 0.0997 & 0.4293 & 0.0924 \\
\hline Back of Hand & 0.3853 & 0.0844 & 0.3712 & 0.0847 \\
\hline Dorsal Surface & 0.4486 & 0.0778 & 0.4381 & 0.0646 \\
\hline Volar Side & 0.3811 & 0.0725 & 0.3702 & 0.0566 \\
\hline All Locations & 0.416 & 0.091 & 0.383 & 0.0839 \\
\hline
\end{tabular}

\section{DISCUSSION}

Human skin signature over the frequency band $80-100 \mathrm{GHz}$ indicate that there is a scatter in emissivity measurements over a range 0.17 to 0.68 , and this is much greater than the experimental measurement uncertainty of \pm 0.002 . Human skin emissivity measurements in Figures 4 and 5 show variations in emissivity from person to person, and at different location on the human body. Estimating the sample mean emissivity values for the 36 males and 24 females separately for all measurements locations indicates the difference between male and female emissivity is $\sim 0.03$. This finding is consistent with the skin of males being thicker than that of females [7]. Experimental measurements of the differences in the emissivity values between the palm of hand and back of hand skin, and between the dorsal and volar regions of the forearm of all 60 participants indicate that the difference in emissivity can be up to 0.074 . This large difference is likely to be due to the much thicker skin on the palm of hand and dorsal area of the forearm. The presence of the blood vessels on the volar forearm and the back of hand locations make the skin more reflective, and as a result human skin emissivity decreases and this results in a significant difference in the emissivity between thinner and thicker skin regions on the body.

Skin thickness and water content are strongly dominating the level of thermal emission emitted from the human body at the millimeter-wave band, and therefore it is recommended for security screening purposes that further measurements are made on larger and more varied groups of individuals, to study how the emissivity varies overall the human body and between individuals. When this is done, expected emissivity values can be identified at each location on the human body and these values can be compared with the measured emissivity from a template ensemble of recognised responses an automated basis at security screening portals. Any deviation from the norm might identify anomalies. This will increase detection probabilities and reduce false alarm rates in the security screening of people. .

Emissivity measurements in this paper are in agreement with the emissivity measurements presented in [4]. These measurements indicate that forearm skin emissivity varies in the range of 0.17 to 0.5 . This range is in agreement with the range of the measured emissivity in this paper (0.17 to 0.68). Emissivity measurements in this paper are also in agreement with the simulated emissivities of the half-space model [8]. 


\section{CONCLUSIONS}

A radiometer effective over the frequency band $80-100 \mathrm{GHz}$ has been investigated and characterised for measuring the human skin signature of a sample of 60 participants. The system was calibrated using liquid nitrogen and ambient temperature sources. These measurements were used to characterise self-emission and millimeter wave radiation from metal plate and fluorescent lights. The mean level of self-emission reflected back from the metal plate was typically \pm 1.0 $\mathrm{K}$ above the background. Millimeter-Wave emission emitted from fluorescent light was found to increase the radiation temperature of the radiometer in the range of $62-74 \mathrm{~K}$ with standard deviation of $\pm 4.0 \mathrm{~K}$.

Radiometric measurements made on a sample of 60 participants show that the emissivity of human skin varies from 0.17 to 0.68 over the $80 \mathrm{GHz}$ to $100 \mathrm{GHz}$ band. Measurements show that the emissivity of the skin varies with the water content and skin thickness. The measurements show that the emissivity of males is higher than that of females by $\sim 0.03$. This supports the knowledge that on average the skin of males is thicker than that of females [7]. Measurements also show the emissivity of thick layers of skin in the human body, such as the palm of the hand and the dorsal forearm was higher than those of the back of the hand and the volar forearm by about 0.0597 to 0.068 for both genders.

Research continues in this area to understand the signature of the human skin at the millimeter wave frequency band to increase detection probabilities and reduce false alarm rates, so ensuring high throughputs at entrances to future airport departure lounges.

\section{FUTURE WORK}

It is recommended that further measurements be made on larger and more varied groups of individuals overall body regions to provide statistics about the emissivity of the human skin. This might be done at a range of frequencies over the millimeter wave frequency bands, the lower frequencies offering greater penetration into under layers of clothing and down to the skin. Whereas, the higher frequencies offering higher resolution. This will lead to greater understanding of human skin signature at the MMW frequency bands and it might be useful for increasing the detection probabilities and reducing the false alarm rate at security screening portals.

\section{REFERENCES}

[1] Salmon, N.A. "Experimental results and simulations from aperture synthesis three-dimensional radiometric imaging", SPIE Millimeter Wave and Terahertz Sensors and Technology IX, SPIE proc. vol. 9993, doi: 10.1117/12.2231696, Edinburgh, September, (2016).

[2] Salmon, N.A. "Characterisation and calibration of a large aperture $(1.6 \mathrm{~m}) \mathrm{ka}$-band indoor passive millimeter wave security screening imager”, SPIE Millimeter Wave and Terahertz Sensors and Technology V, SPIE proc. vol. 8544, doi: 10.1117/12.999278, Edinburgh, September, (2012).

[3] Pozar D.M., [Microwave Engineering], John Wiley \& Sons Publisher, New Jersey \& Hoboken, 497-511 (2011).

[4] Harmer, S.W., Shylo, S., Shah, M., Bowring, N.J. and Owda, A.Y., "On the feasibility of assessing burn wound healing without removal of dressings using radiometric millimeter-wave sensing," Progress In Electromagnetics Research. Papers 45,173-183 (2016).

[5] Gray, H., [Anatomy of the Human Body], Lea \& Febiger Publisher, United States \& Philadelphia, 1320-1330 (1918).

[6] Millington, P.F., Wilkinson, R., [Skin (Biological Structure and Function)], Cambridge University Press Publisher, United Kingdom \& Cambridge, 48-80 (2009).

[7] Diridollou, S.,Vabre, V., Berson, M.,Vaillant, L., Black, D., Lagarde, J.M. , Grégoire, J.M., Gall,Y. and Patat, F., "Skin ageing: changes of physical properties of human skin in vivo," International Journal of Cosmetics Science. Papers 23, 353-362 (2001).

[8] Owda, A.Y., Salmon, N.A., Harmer, S.W., Shylo, S., Bowring, N.J., Rezgui, N.D. and Shah, M., "Millimeter-Wave Emissivity as a Metric for the Non-Contact Diagnosis of Human Skin Conditions", Journal of Bioelectromagnetics, vol. 38, issue, 7, pp. 559-569 (2017). 